\title{
Purine Nucleoside Phosphorylase (PNP) Deficiency: A Case Report Rawia Albar ${ }^{1}$, Alaa Alesa ${ }^{2}$, Dina Saqa ${ }^{3}$, Jamanah Abdulaziz ${ }^{2}$, Mohammad Batouk ${ }^{4}$, Abdullah Alesa ${ }^{1}$ \\ ${ }^{1}$ King Abdulaziz Medical City, Jeddah, ${ }^{2}$ King Saud bin Abdulaziz University for Health Sciences College of Medicine, ${ }^{3}$ Ibn Sina National College for Medical Studies, ${ }^{4}$ King Abdulaziz University. Corresponding Author: Alaa Alesa, E-Mail: alaa.alesa@gmail.com, Phone : +966583399699
}

\begin{abstract}
Background: Purine nucleoside phosphorylase (PNP) deficiency is a rare genetic disease that results in combined immunodeficiency. Its inheritance is autosomal recessive and affects the purine metabolic pathway. There is a profound effect on T-cells and variable B-cell dysfunction. There is a strong association with neurological dysfunction in as many as two thirds of cases and autoimmunity in one third. When PNP activity is absent or greatly diminished, deoxyguanosine triphosphate (dGTP) is believed to accumulate in the mitochondria, which inhibits ribonucleotide reductase and mitochondrial DNA repair. This is very harmful to T lymphocytes and leads to DNA damage and apoptosis during thymus selection.

Case: A toddler girl diagnosed with PNP) deficiency that is currently being managed using different type of modalities with good response.

Results: The patient was admitted in an outside hospital when she developed fever for 4 days along with a perianal abscess. Septic shock was suspected, and she was initially started on gentamycin and tazocin then switched to vancomycin, mereponem and metrodinazole. After resolution of fever, the patient was transferred to our tertiary care center to undergo immunology work up. Upon finally receiving the patient she was stable and afebrile, however there was severe neutropenia and leukopenia. A multidisciplinary team, which included general pediatrics, hematology, Infectious diseases and immunology, handled her case. Furthermore, the patient was switched back to tazocin and gentamycin and completed 14 days with good response and full resolution of fever. Following the multidisciplinary team plan, an immunology/hematology work up was initiated.

Conclusion: After 14 days of treatment, there was a good response and resolution of fever. Then, a multidisciplinary team plan, an immunology/hematology work up was initiated.
\end{abstract}

Keywords: PNP, immune deficiency, immunity, pediatrics.

\section{INTRODUCTION}

Purine nucleoside phosphorylase (PNP) deficiency is a rare genetic disease that results in combined immunodeficiency. Its inheritance is autosomal recessive and affects the purine metabolic pathway. There is a profound effect on T-cells and variable B-cell dysfunction. There is a strong association with neurological dysfunction in as many as two thirds of cases and autoimmunity in one third ${ }^{(1,2)}$.

Giblett et al. ${ }^{(3)}$ was the first to describe PNP deficiency in 1975 shortly after discovering a deficiency of another enzyme of the purine metabolic pathway, adenosine deaminase (ADA). When PNP activity is absent or greatly diminished, deoxyguanosine triphosphate (dGTP) is believed to accumulate in the mitochondria, which inhibits ribonucleotide reductase and mitochondrial DNA repair. This is very harmful to T lymphocytes and leads to DNA damage and apoptosis during thymus selection. There are many disease-causing mutations in the PNP gene and approximately 17 have been reported to date, coupled with the somewhat variable course of the disease associated with PNP deficiency that may be related to this genetic heterogeneity ${ }^{(4)}$.
We presented a case of a thirty-three month old girl diagnosed with PNP deficiency who had multiple infections requiring hospitalizations along with hypotonia and global developmental delay.

\section{Case description:}

This is a Thirty-three month old, full term female child from Saudi Arabia. She was delivered by spontaneous vaginal delivery with no perinatal complications to report. The parents are $1^{\text {st }}$ degree relatives and the mother is a primigravida with no history of abortions.

However, the child was known to have autosomal recessive immunodeficiency due to purine nucleoside phosphorylase deficiency.

There were central hypotonia and a history of recurrent oral thrush. Moreover, there was a history of one previous hospitalization before at the age of 9 months due to a respiratory infection. Recently she was admitted in an outside hospital when she developed fever for 4 days along with a perianal abscess. Septic shock was suspected, and she was initially started on gentamycin and tazocin then switched to vancomycin, mereponem and 
metrodinazole. After resolution of fever, the patient was transferred to our tertiary care center to undergo immunology work up. Upon finally receiving the patient she was stable and afebrile, however there was severe neutropenia and leukopenia.

A multidisciplinary team, which included general pediatrics, hematology, Infectious disease and immunology, handled her case. Furthermore, the patient was switched back to tazocin and gentamycin and completed 14 days with good response and full resolution of fever. Following the multidisciplinary team plan, an immunology/hematology work up was initiated. The lymphocytes markers are listed in the table (1).

Bond marrow aspiration showed no blast or abnormal cells. No hemophagocytes seen. Furthermore, granulopoiesis was reduced and it showed maturation arrest of neutrophils at promyelocytes stage with no dysplasia. Chest X-ray showed clear lung fields with no opacity, no pleural effusion and no pneumothorax. No acid-fast bacilli was seen in the smear and blood culture showed no growth. Finally, the inflammatory markers were within normal limits. Upon reviewing her labs and taking into consideration her good response to the antibiotics, she was discharged home in good stable condition with follow up in immunology clinic after one week.

The patient was seen in the outpatient clinic for review and follow up. The plan was to repeat her labs including IgG levels and planed to administer IVIG if levels are found to be reduced. Finally, the family were planned to be tested for HLA typing for possible bone marrow transplantation.

Table (1): Lymphocyte Markers

\begin{tabular}{|l|l|l|}
\hline \multicolumn{1}{|c|}{ Lymphocyte Markers } & \multicolumn{1}{c|}{ Results } & \multicolumn{1}{c|}{ Normal Range } \\
\hline CD3+[T-Cell] & $118.00 \mathrm{cells} / \mathrm{mcL} \mathrm{L}$ & {$[1800.00-3000.00]$} \\
\hline CD3+CD4+[T-Helper] & $83.00 \mathrm{cell} / \mathrm{s} / \mathrm{mLL} \mathrm{L}$ & {$[1000.00-1800.00]$} \\
\hline CD3+CD8+[T-Supp] & $1.0 \mathrm{cells} / \mathrm{mcL} \mathrm{L}$ & {$[800.00-1500.00]$} \\
\hline CD19+[B-Cell] & $29.0 \mathrm{cells} / \mathrm{mcL} \mathrm{L}$ & {$[700.0-1300.0]$} \\
\hline Cd16+65+[NK] & $89.0 \mathrm{cell} / \mathrm{s} / \mathrm{mcL} \mathrm{L}$ & {$[200.0-600.0]$} \\
\hline Lymph & $20.10 \% \mathrm{~L}$ & {$[38.00-53.00]$} \\
\hline CD4/CD8 Ratio & $7.57 \mathrm{H}$ & {$[1.00-1.60]$} \\
\hline WBC & $1.3 \times 10^{\wedge} 3 / \mathrm{uL}$ & \\
\hline
\end{tabular}




\section{DISCUSSION}

Purine nucleoside phosphorylase PNP deficiency is a rare disease accounting for $4 \%$ of all severe combined immunodeficiency (SCID) cases worldwide and relatively few cases have been described in the literature especially in the Middle East ${ }^{(5)}$. Furthermore, this deficiency is an autosomal recessive disease caused by mutations in PNP gene. This mutation results in a defect of PNP enzyme activity, which is important in the process of degradation of purine nucleosides ${ }^{(6)}$. This in turn, causes a build up of a toxic substance known as deoxyguanosine triphosphate (dGTP), which is very harmful especially to premature lymphocytes and therefore causing a shortage in Blymphocytes and $\mathrm{T}$ lymphocytes. This effect on lymphocytes gives rise to the combined immunodeficiency. Hence, exposing the patient to recurrent infections and opportunistic infections, which can often be life threatening. Many cases present with multiple infections and hospitalizations before diagnosing PNP deficiency. Our patient had 2 hospitalizations before the age of 3 years and a recurrent oral thrush, which was explained by the immune deficiency that she has. Moreover, the build up of (dGTP) is thought to be the reason in many patients who have PNP deficiency and have neurological involvement because of the toxic build up of (dGTP). This is also very similar to our case where we noted global developmental delay and central hypotonia. In addition, these patients are at a higher risk of acquiring autoimmune diseases like; autoimmune hemolytic anemia 'the commonest', autoimmune neutropenia, idiopathic thrombocytopenic purpura (ITP), systemic lupus and thyroiditis. Other manifestations of PNP deficiency include developmental delay, intellectual disability, ataxia and spasticity ${ }^{(7)}$. These neurological manifestations account for the majority of the patients' main presentation talking about two thirds of them. Low levels of serum uric acid may indicate the diagnosis as it could be normal in some of the patients, so the diagnosis can be supported by reduced PNP activity and established by molecular gene analysis ${ }^{(8)}$. Reviewing the results of a study by La Marca et al. ${ }^{(9)}$ where they reported that Tandem mass spectrometry (TMS) is applicable method for diagnosis and screening of PNP deficiency on dried blood spots (DBSs) of affected patients with minimal cost. Whereas, T cell-receptor excision circle (TREC) or Kappa-deleting recombination excision circle (KREC) measurement alone could be misleading ${ }^{(9)}$. Hematopoietic stem cell transplantation (HSCT) remains the only cure for many immunodeficiencies including PNP deficiency. Gene therapy could be a better choice for treatment that does not require the presence of a donor ${ }^{(10)}$. PNP deficiency, if left untreated, rapidly causes death due to repeated infections within the first or second decade of life. Bone marrow transplant treatment can effectively treat and improve the prognosis of problems with the immune system. Unfortunately, the neurological problems associated with this disease will not be corrected by bone marrow transplantation ${ }^{(11)}$.

\section{CONCLUSION}

In conclusion, Purine nucleoside phosphorylase (PNP) deficiency is a rare disease. Management of patients with this type of conditions is very challenging. A multidisciplinary team approach is vital to successfully manage such complex cases. Multiple treatment modalities have been utilized in treatment of such cases. However, very few are successful. In the future, we aspire that there will be better and more successful treatment modalities available to treat such complex cases.

\section{REFERENCES}

1. Markert M (1991): Purine nucleoside phosphorylase deficiency. Immunodeficiency reviews, 3 (1): 45-81.

2. Myers L, Hershfield M, Neale W, Escolar M, Kurtzberg J (2004): Purine nucleoside phosphorylase deficiency (PNP-def) presenting with lymphopenia and developmental delay: successful correction with umbilical cord blood transplantation. The Journal of pediatrics, 145 (5): 710-2.

3. Giblett E, Ammann A, Sandman R, Wara D, Diamond L (1975 ): Nucleoside-phosphorylase deficiency in a child with severely defective T-cell immunity and normal B-cell immunity. The Lancet, 305 (7914): 1010-3.

4. Grunebaum E, Zhang J, Roifman C (2004): Novel mutations and hot- spots in patients with purine nucleoside phosphorylase deficiency. Nucleosides, Nucleotides and Nucleic Acids, 23 (8-9): 1411-5.

5. Markert M (1991): Purine nucleoside phosphorylase deficiency. Immunodeficiency reviews, 3 (1): 45-81.

6. Madkaikar MR, Kulkarni S, Utage P, Fairbanks L, Ghosh K, Marinaki A, Desai M (2011): Purine nucleoside phosphorylase deficiency with a novel PNP gene mutation: a first case report from India. https://pubmed.ncbi.nlm.nih.gov/22669887/

7. Simmonds H, Fairbanks L, Morris G, Morgan G, Watson A, Timms P, Singh B (1987): Central nervous system dysfunction and erythrocyte guanosine triphosphate depletion in purine nucleoside phosphorylase deficiency. Archives of disease in childhood, 62 (4): 385-91.

8. Robak T, Lech-Maranda E, Korycka A, Robak E (2006): Purine nucleoside analogs as immunosuppressive and antineoplastic agents: mechanism of action and clinical activity. Current medicinal chemistry, 13 (26): 3165-89.

9. La Marca G, Canessa C, Giocaliere E et al. (2014): Diagnosis of immunodeficiency caused by a purine nucleoside phosphorylase defect by using tandem mass spectrometry on dried blood spots. Journal of allergy and clinical immunology, 134 (1): 155-9.

10. Vong L (2018): Gene therapy for PNP deficiency protocol. Lymphosign Journal, 5 (3): 115-20.

Broome C, Graham M, Saulsbury F et al. (1996): Correction of purine nucleoside phosphorylase deficiency by transplantation of allogeneic bone marrow from a sibling. The Journal of pediatrics, 128 (3): 373-6. 